\title{
Market Forces and Price Ceilings: A Classroom Experiment
}

Jamie Brown Kruse, Ozlem Ozdemir and Mark A. Thompson

\begin{abstract}
The effect of price controls on competitive equilibrium is a standard topic in many undergraduate economics courses. This classroom experiment demonstrates the effect of rent control (price ceilings) on the market for apartments. As participants in the experiment, students experience the effect of a price ceiling as buyers (renters) and sellers (landlords). The classroom-posted offer market exhibits a shortage under a binding price ceiling. Further, we explore a secondary response to rent control. When given the opportunity, landlords lower the quality of the apartments by reducing maintenance expenditures under the price ceiling, thus moving the market back to equilibrium. Since many students are themselves renters, they should relate to changes in quality due to lower maintenance by landlords. This experiment will stimulate discussion on market forces and on public policy aimed at restricting prices.
\end{abstract}

In many cases rent control appears to be the most efficient technique presently known to destroy a city - except for bombing. (Lindbeck, 1972, p. 39)

\section{Introduction}

This paper describes a simple classroom experiment that demonstrates the effect of price controls and the equilibration process. Competitive equilibrium is a foundation of neoclassical economics and the imposition of binding price controls is often used as the first illustration of market failure. The topic is dealt with in early chapters of many intermediate economics textbooks (e.g. Browning and Zupan, 1999; Mansfield and Yohe, 2000; Perloff, 1999; Varian, 1999). In addition, O'Sullivan and Seffrin (2001) and Sharp, Register and Grimes (2002) are examples of texts for less advanced courses that discuss price controls and secondary responses. 
In spite of the thorough discussion in the texts, we suspect that many students do not fully appreciate the meaning of deadweight loss or the forces that drive market equilibrium. A classroom experiment offers a different perspective that can enrich student understanding. The student, as a participant within a classroom experiment, can see prices and quantities unfold, can experience gains from trade, and can observe the dynamics that carry the market back to a new equilibrium after the imposition of a price restriction. The classroom experiment we describe here investigates the effect of price ceilings using the context of an apartment rental market. A price ceiling in the form of rent control is introduced that reduces market efficiency. The last phase of the experiment allows market forces acting through a quality variable to equilibrate supply and demand. The deterioration in quality of the product in our experimental market is similar to the description by Stiglitz (2000) of 'quality adjustments' made by landlords in response to rent control. In order to assess student understanding of and attitudes towards rent control, we used a short survey that was administered before as well as after the classroom market. The survey results demonstrate that a classroom experiment can be an effective pedagogical method. ${ }^{1}$

This experiment gives students a more dynamic perspective on the response of market forces to constraints on the system. While this paper describes a simple posted-offer market with price controls, it does not explore novel territory. Research-oriented laboratory experiments that explored price controls were published over 20 years ago (e.g. Isaac and Plott, 1981; Smith and Williams, 1981). One contribution of this paper lies in its departure from what are considered standard controlled experimental methods such as private information and minimal use of context. The reason for the departure is to make this experiment a richer and more effective pedagogical tool. Those who are familiar with laboratory experiments will recognise our movement away from the controlled research experiment - we ask participants in the experiment who are affected by a shortage to raise their hands so that everyone sees the immediate impact of a shortage. We allow controlled discussion during the experiment. We purposefully selected a context-rich familiar scenario so that students could see the applicability of simple economic models to their day-to-day lives. As classroom experiments evolve as important and engaging pedagogical tools, we think that a separate set of criteria will emerge that identify properties of a well-designed classroom experiment in much the same way that Smith (1982) laid out the characteristics of a welldesigned controlled laboratory experiment for research purposes.

This classroom experiment is appropriate for introductory principles courses, intermediate-level courses, and undergraduate field courses such as managerial economics and public finance. The experiment can be adjusted for varying class sizes. 
The hand-run version we describe here works best in classes or recitation sections of fewer than 40 students. The remainder of the paper is organised as follows: We describe the procedure for the classroom experiment in detail in the next section. We follow this with a description of the theoretical predictions for all three phases of the experiment and discuss our survey that accompanied the experiment, conducted with students at Texas Tech University. The final section contains a discussion of the characteristics of an effective pedagogical experiment and our concluding remarks.

\section{Procedure}

In this section, we will start with a short summary of the experiment, which uses a posted-offer trading mechanism, and follow it with a detailed description of the procedures. To begin with, students are given the scenario in which they will play the role of either a landlord or a renter in a fictitious apartment market. The market has several trading periods. The initial periods are devoted to unrestricted transactions, where the participants become accustomed to their roles and the market converges towards a competitive equilibrium. After about four trading periods, a binding price ceiling (rent control) is imposed. Two periods of trade under rent control are usually sufficient to make students aware of excess demand (shortage) conditions. In the latter part of the experiment, we allow landlords to adjust their maintenance expenditures, thereby reducing the quality of the apartment. ${ }^{2}$ Students will see that when price is constrained, the market will attempt to equilibrate through adjustments in other variables that affect demand and supply - in this case, quality. ${ }^{3}$

The classroom experiment begins with the distribution of a one-page instruction and record sheet that assigns each participant the role of either renter (Figure 1a), with a rental budget in each period, or landlord (Figure 1b), with a cost each period. A complete set of rental budgets and landlord costs for a 20-participant experiment can be found in the appendix. These instructions mainly describe the incentive structure for renters and landlords. ${ }^{4}$ After the record sheets have been distributed and read, verbal instructions are given describing the posted-offer market. Using the protocol for this trading institution, landlords (sellers) must make the first decision. Each landlord, using his or her own private information about the cost of maintaining the apartment, is instructed to write on a message slip the rental price they are willing to accept for the apartment. After message slips have been collected from all landlords, the rental prices are 'posted' on the blackboard alongside their respective apartment ID numbers.

All renters (buyers) can see all prices that are posted. Renters are allowed to make their buying decision sequentially. ${ }^{5}$ When it is a renter's turn, he or she can select 
Figure 1a. Instruction and record sheet: renters

\section{Renter identification number: XX}

In this market you are a renter. You can either rent an apartment or live with your obnoxious relatives. The column called 'Rental budget' states how much money you have to spend on rent. If you rent an apartment for less than your rental budget, then you have extra money to spend on other things. If you don't rent an apartment, you must pay your relatives the amount of your rental budget to live with them. Keep track of how much money you saved from your rental budget. You will get bonus points for how much money you saved over the entire class period.

\begin{tabular}{|c|c|c|c|}
\hline Period & Rental budget (\$) & Apartment \# and rental price (\$) & Money saved \\
\hline 1 & 1000 & & \\
\hline 2 & 550 & & \\
\hline 3 & 750 & & \\
\hline 4 & 850 & & \\
\hline 5 & 1000 & & \\
\hline 6 & 550 & & \\
\hline 7 & 750 & & \\
\hline 8 & 850 & & \\
\hline
\end{tabular}

Your total

Your name

Student ID

among the prices for apartments that are available. Presumably, each renter will pick the lowest price available that is higher than his or her reservation value (rental budget on the instruction sheet). If the renter picks an available price, a line is drawn through the price and apartment number on the board to indicate that it is no longer available. The renter can choose not to rent an apartment, in which case he or she says 'Pass' to reject all prices.

Once an apartment has been chosen, the renter must record the rental price and the apartment ID number on his or her sheet. The renter's ID number is written on the landlord's message sheet and returned to the landlord. This process continues, giving each renter a turn until all renters have had the opportunity to choose, thus concluding a trading period. The instructor asks if anyone has any questions. 
Figure 1b. Instruction and record sheet: landlords

\section{Landlord identification number: XX}

In this market you are a landlord. You own one apartment. The column called 'Rental cost' states how much money the apartment costs you to maintain when you have a renter. As long as your rental price is more than the rental cost, you make money. If you don't have a renter, your rental cost is zero but you don't make any money either. Keep track of how much money you make because you will get bonus points for how much money you made during the entire class period.

\begin{tabular}{|c|c|c|c|}
\hline Period & Rental price (\$) & Rental cost (\$) & Money made (\$) \\
\hline 1 & & 525 & \\
\hline 2 & & 475 & \\
\hline 3 & & 275 & \\
\hline 4 & & 725 & \\
\hline 5 & & 525 & \\
\hline 6 & & 475 & \\
\hline 7 & & 275 & \\
\hline \multirow[t]{4}{*}{8} & & 725 & \\
\hline & & Your total & \\
\hline & & Your name & \\
\hline & & Student ID & \\
\hline
\end{tabular}

If there are none, the next trading period begins. ${ }^{6}$

The first four periods are dedicated to free-market adjustment with no price controls; we can call this the free market phase. Four periods are usually sufficient for students to become familiar with the protocol and see the price formation process as the classroom market moves towards equilibrium. Figure 2 shows the results from a classroom session conducted with 20 participants at Texas Tech University. The transaction prices for each period are indicated by dots. The total quantity traded is listed just above the trading period. Notice that for the first four trading periods there is considerable price dispersion, with prices ranging from $\$ 350$ to $\$ 700$ by period 4 . Also note that the quantity traded was $9,8,8$ and 7 for periods $1-4$ respectively. The competitive quantity for the parameter set we used is 8 units. 
Figure 2. Apartment prices and transactions by trading period

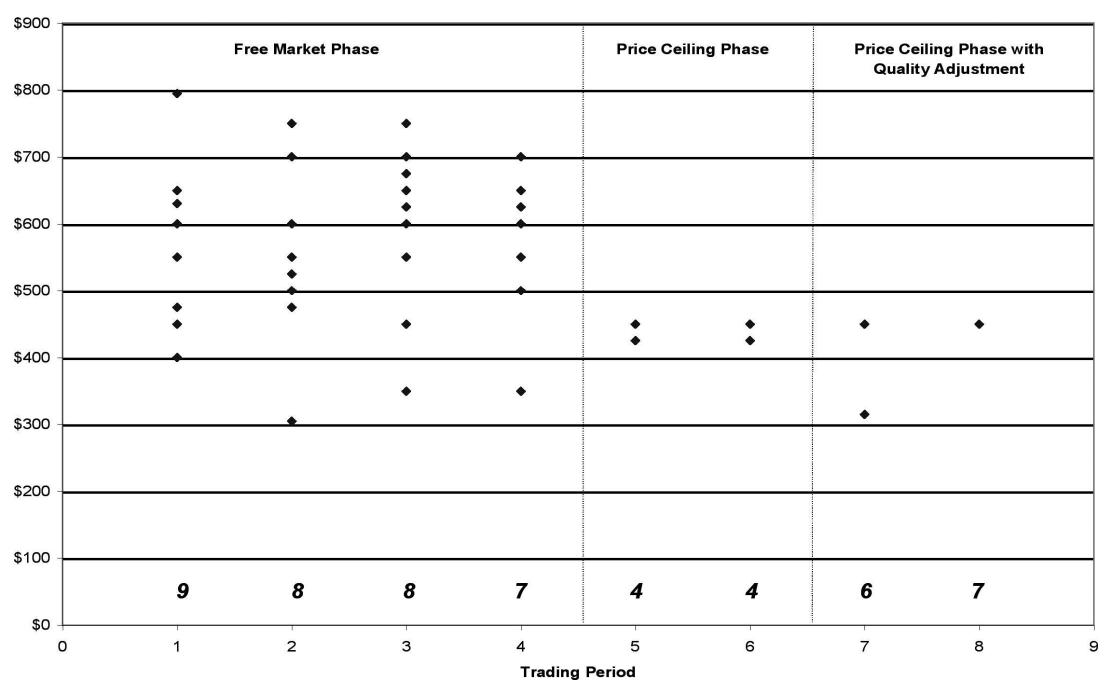

Notes: The dots represent the different prices posted by the landlords. The number above the trading period (in italics) indicates the number of apartment transactions. Free market phase: the equilibrium price and quantity; $\mathrm{P}=[\$ 625, \$ 650], \mathrm{Q}=8$. Price ceiling phase: the price and quantity; $\mathrm{P}=\$ 450$ (price ceiling), $\mathrm{Q}=4$. The excess demand (shortage of apartments) due to the price ceiling is 6 . Price ceiling phase with quality adjustment: the equilibrium price and quantity; $\mathrm{P}=\$ 450$ (price ceiling), $\mathrm{Q}=7$.

After the completion of the free market phase, we enter the price ceiling phase. At the start of fifth period, we announce that the landlords can only state selling prices of $\$ 450$ or less. We ask if any landlord wants to offer a rental price less than $\$ 450$ and note the rental prices (if any) below $\$ 450$ on the board along with the apartment ID. We then ask for a show of hands for landlords who want to offer exactly $\$ 450$ and then list their apartment ID numbers on the blackboard, each with the rental price of $\$ 450$. Again, the renters are queued up to make their apartment decisions sequentially. With the landlord costs and rental budgets that we used, the rent control will create a shortage. Only four landlords have low enough costs that the $\$ 450$ price ceiling is profitable, whereas ten renters are willing to rent an apartment at that price. This, of course, means that there will be no apartments left before the queue is exhausted. To illustrate the shortage, we ask the renters that were willing to pay $\$ 450$ but didn't get an apartment to hold up their hands. In the next period (period 6), we repeat the price ceiling protocol. 
The results of the price ceiling phase can be seen for periods 5 and 6 in Figure 2 . The total quantity of four traded in each period at prices ranging from $\$ 425$ to $\$ 450$.

The seventh period marks the beginning of the quality adjustment phase. We allow the landlords to reduce maintenance expenditures on the apartment (i.e.quality adjustment). The class will see how market forces respond to the price restriction. At the beginning of the period, we announce that the rent control is still set at $\$ 450$; however, the landlords can reduce the amount they spend on maintaining the apartment by $\$ 150$. We then repeat the process of asking for rental prices and apartment ID numbers, as in periods 5 and 6 . In addition, we ask the landlords to state whether they have chosen a high or low level of maintenance for their apartment (a low level of maintenance reduces landlord costs by $\$ 150$ ). We tell renters that if an apartment is the 'low maintenance' kind, the value of the apartment is now $\$ 150$ less because they must spend $\$ 150$ to provide some maintenance and amenities that the landlord no longer provides. We then allow renters to choose the apartments as in prior trading periods.

We repeat the process for the final trading period (period 8). Students are then instructed to sum up their earnings and write their names on the record sheet. The results for trading periods 7 and 8 shown in Figure 2 show the market readjusting. Notice that, by the last period, seven apartments were being traded for exactly $\$ 450$, which is the competitive equilibrium prediction for the market with quality adjustment. A more detailed discussion of the demand and supply curves and the competitive predictions follows in the next section.

\section{Discussion}

The main idea of the experiment relies on the basic tenets of a market described by Adam Smith (1776). Each agent, acting in his or her own self-interest, provides the nascent conditions for an efficient market. A price restriction in the form of rent control reduces the ability of the agents to extract the potential gains from trade. The first six periods of this experiment are sufficient to demonstrate the loss in allocative efficiency caused by binding price controls. We elected to use the final two periods to show how the market might adjust to the price restriction. At the conclusion of the experiment, we show students the competitive equilibrium price and quantity predicted for the supply and demand schedules created in the class. This can be done by polling all the landlords and asking what their cost was for the first period and arranging the costs from lowest to highest, thereby creating the supply schedule. Then renters are asked to report their rental budgets for the first period. By arranging the rental budgets from highest to lowest, we have a demand schedule. 
Figure 3a. Discrete demand and supply functions: periods 1-4

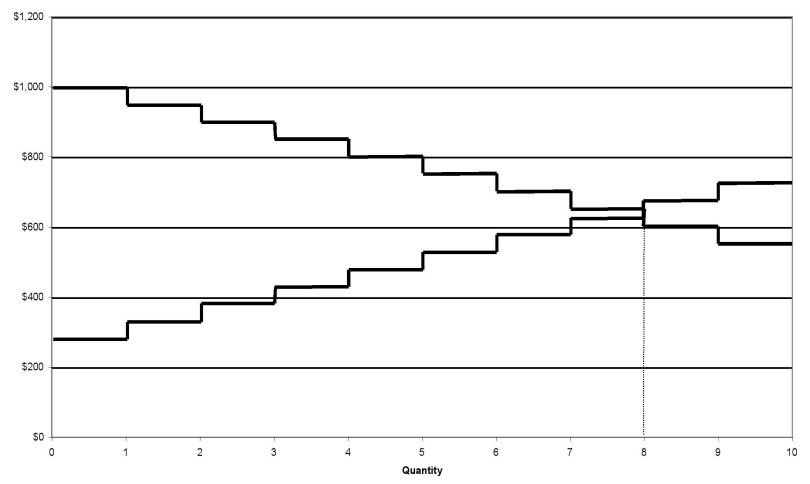

Notes: The equilibrium price and quantity: $P=[\$ 625, \$ 650], Q=8$. The equilibrium price is not unique since any trading price between $\$ 625$ and $\$ 650$ will maximise gains from trade with 8 units traded.

Figure 3b. Discrete demand and supply functions with price ceiling: periods 5 and 6

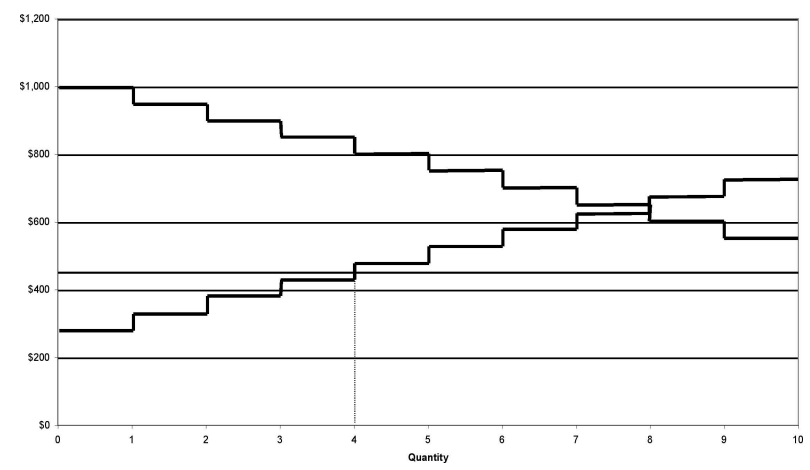

Notes: $P=\$ 450$ (price ceiling), $Q=4$. The excess demand (shortage of apartments) due to the price ceiling is 6 .

Figures $3 a, 3 b$ and $3 c$ graph the demand and supply schedules using our parameters for the free market phase, price ceiling phase and price ceiling-plusquality-adjustment phase respectively. ${ }^{7}$ The schedule of rental budgets (demand) 
Figure 3c. Discrete demand and supply functions with quality adjustment: periods 7 and 8

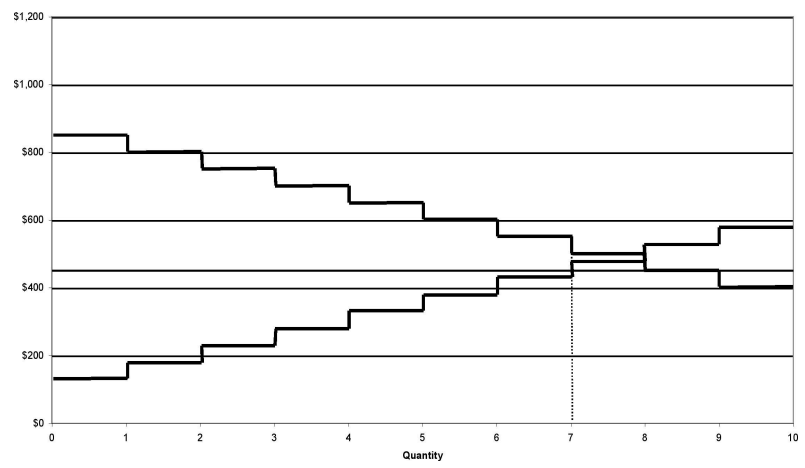

Note: The equilibrium price and quantity: $P=\$ 450$ (price ceiling), $Q=7$.

and landlord costs (supply) for 20 students are in the appendix. In the class discussion of results, we draw the demand and supply curves for the free market phase. Next, we draw in the price ceiling of $\$ 450$ and comment on how many apartments were available at that price and how many people wanted an apartment. Here are some discussion points we often use:

- Ask the students what was the amount of the shortage. Were there students who wanted an apartment, yet were unable to get one?

- Ask students if rent control helped the people with the lower rental budgets get an apartment. If low-value renters happened to get lucky, one or two might get an apartment, but since the quantity offered on the market goes down, it actually gets harder to find an apartment.

- Discuss the seventh and eighth periods as a natural response to the price ceiling. How many landlords lowered their cost by choosing to reduce apartment maintenance?

In order to assess the understanding and attitudes toward price restrictions on apartments, we asked three survey questions prior to the experiment in a managerial economics class at Texas Tech University with 30 students; 20 of the students were active participants and the remaining 10 were observers. The results are given in Table 1. 
Table 1. Survey questions $(N=30)$

1 (a) Do you (currently) rent an apartment?

Before experiment

Yes: $77 \% \quad$ No: $23 \%$

(b) Have you ever rented an apartment?

Before experiment

Yes: $97 \% \quad$ No: $3 \%$

2 Would you encourage the government to apply a price ceiling (rent control) to the apartment market?
Before experiment
Yes: $60 \%$
No: $40 \%$

After experiment

Yes: $23 \%$

No: $77 \%$

3 If an effective price ceiling is placed on the apartment market, do you believe that the quality of the apartments will be negatively affected?
Before experiment
Yes: $97 \%$
No: $3 \%$
After experiment
Yes: $97 \%$
No: $3 \%$

Note that prior to the experiment, in response to question 2,'Would you encourage the government to apply a price ceiling (rent control) to an apartment market?', a relatively large proportion (60\%) of the students answered yes, in spite of their indication that they understood the probable effect of a price ceiling on quality (see question 3). We want to point out that these results are from an advanced economics class where all the students have taken an economic principles course.

After the classroom experiment, we asked questions 2 and 3 again. As a result of the experiment, students had experienced the problems created by the price ceiling. Many students who witnessed the effect of the price ceiling on the market changed their mind on question 2. The proportion that supported governmentimposed price ceilings on apartments went from $60 \%$ before to $23 \%$ after the classroom experiment.

Recall that the survey was administered to all 30 students in the course - 20 who participated in the experiment and 10 who observed. There were no qualitative differences between the survey responses of the 10 observers and the 20 participants. Evidently, the effect of a regulatory policy such as a price ceiling becomes more salient when observed in an experimental setting. The results of the survey questions lend support to the conclusion that the classroom experiment is an effective pedagogical device to help students understand market mechanisms and price restrictions. 
Most students believed the effective price ceiling would be beneficial since they would pay a lower price for the apartment (but they assumed they would get an apartment). However, the students responded to the experiment by recognising that a lower price (i.e. price restriction by the government) has a trade-off. First, the price ceiling will create a shortage of apartments. Second and due to the shortage, market forces will attempt to equilibrate supply and demand for apartments in some other way (i.e.quality adjustments in this case). The end result that the students learned is that the price restriction only made the 'situation worse.' As one student stated,'I did not expect the price ceiling (which I knew would cause a shortage) to affect me ... I figured I could still get an apartment, just at a lower price!'

\section{Concluding remarks}

Smith (1982) described five sufficient conditions for a controlled laboratory experiment. ${ }^{8}$ They are: nonsatiation, saliency, dominance, privacy and parallelism. For pedagogical purposes, factors that enhance learning dominate the need for precise laboratory control. We will not go so far as to state precepts of a classroom experiment, but we do offer characteristics that define the suitability of an experiment for the classroom. Holt (1999) also discusses desirable factors important in classroom experiments. A pedagogical experiment should be:

- Transparent. An effective experiment provides a clear connection between the concept and experiment that is easy to see by observing the public information. Rich context can help students understand the structure of the institution as well as its relevance to real-life decisions.

- Manageable. The experiment should be designed so that ideally a single person can conduct the exercise. This means that the procedure of collecting and processing information from participants should be kept to a minimum. In the experiment reported here, we collect landlord rental prices on message sheets to enforce simultaneous decisions. Otherwise, most interaction is verbal and posted on the board for all to see.

- Scaleable. An experiment is more useful if it can accommodate different class sizes. Individual choice experiments are easiest to scale to class size. Experiments that rely on aggregation are more challenging. The parameter set we provide in the appendix is designed for exactly 20 participants. The size of the market can be adjusted by changing the slope and intercept terms of the supply and demand functions on which they are based.

- Brief.Class time is limited and valuable. Whereas many research experiments require 1-3 hours or even multiple meetings, a classroom experiment plus 
discussion should fit into a 50-minute class period. In our experience, the classroom market reported here with 8 market periods takes approximately 30 minutes to complete.

Classroom experiments can serve as a pedagogical tool to enhance students' learning. The goal of the classroom experiment is for students to understand their decision environment and how decisions affect personal laboratory earnings (reward) as well as to notice and appreciate the effect of self-interested behaviour on the aggregate (market) outcome. Thus, this experiment not only stimulates discussion on market forces and on public policy aimed at restricting prices, but also gives students a chance to experience possible real-life applications of textbook knowledge. In addition, this experiment may serve as a starting point to initiate further discussion on public policy issues related to government regulation.

Appendix: Example rental budgets and landlord costs for a 20-participant market

Renters

\begin{tabular}{rrrrrrrrrrrr}
\hline $\begin{array}{l}\text { Period } \\
\text { R1 }\end{array}$ & R2 & R3 & R4 & R5 & R6 & R7 & R8 & R9 & \multicolumn{2}{c}{ R10 } \\
\hline 1 & 1000 & 950 & 900 & 850 & 800 & 750 & 700 & 650 & 600 & 550 \\
2 & 550 & 600 & 650 & 700 & 750 & 800 & 850 & 900 & 950 & 1000 \\
3 & 750 & 700 & 600 & 550 & 1000 & 950 & 900 & 850 & 800 & 750 \\
4 & 850 & 900 & 950 & 1000 & 550 & 600 & 650 & 700 & 750 & 800 \\
5 & 1000 & 950 & 900 & 850 & 800 & 750 & 700 & 650 & 600 & 550 \\
6 & 550 & 600 & 650 & 700 & 750 & 800 & 850 & 900 & 950 & 1000 \\
7 & 750 & 700 & 600 & 550 & 1000 & 950 & 900 & 850 & 800 & 750 \\
8 & 850 & 900 & 950 & 1000 & 550 & 600 & 650 & 700 & 750 & 800 \\
\hline
\end{tabular}

\section{Landlords}

\begin{tabular}{lllllllllllll}
\hline $\begin{array}{l}\text { Period } \\
\text { L1 }\end{array}$ & L2 & L3 & L4 & L5 & L6 & L7 & L8 & L9 & \multicolumn{1}{c}{ L10 } \\
\hline 1 & 275 & 325 & 375 & 425 & 475 & 525 & 575 & 625 & 675 & 725 \\
2 & 725 & 675 & 625 & 575 & 525 & 475 & 425 & 375 & 325 & 275 \\
3 & 525 & 575 & 625 & 675 & 725 & 275 & 325 & 375 & 425 & 475 \\
4 & 475 & 425 & 375 & 325 & 275 & 725 & 675 & 625 & 575 & 525 \\
5 & 275 & 325 & 375 & 425 & 475 & 525 & 575 & 625 & 675 & 725 \\
6 & 725 & 675 & 625 & 575 & 525 & 475 & 425 & 375 & 325 & 275 \\
7 & 525 & 575 & 625 & 675 & 725 & 275 & 325 & 375 & 425 & 475 \\
8 & 475 & 425 & 375 & 325 & 275 & 725 & 675 & 625 & 575 & 525 \\
\hline
\end{tabular}


Notes: The reservation prices and costs in the tables above were generated using the discrete units inverse demand function, $P=1050-50 \mathrm{Q}$ and inverse supply function, $P=225+50 \mathrm{Q}$. Other parameter sets can be generated that accommodate more or fewer participants by adjusting the intercepts and/or slope terms of the demand and supply functions.

\section{References}

Browning, E. K. and Zupan, M. A. (1999) Microeconomic Theory and Applications, 6th edn, Reading, MA: Addison Wesley. Davis, D. and Holt C. (1993) Experimental Economics, Princeton, NJ: Princeton University Press.

DeYoung, R. (1993) 'Market experiments: the laboratory versus the classroom', Journal of Economic Education, vol. 24, pp. 335-51.

Gyourko, J. and Linneman, P. (1990) 'Rent controls and rental housing quality: a note on the effects of New York City's old controls', Journal of Urban Economics, vol. 27, pp. 398-409.

Holt, C. A. (1999) 'Teaching economics with classroom experiments', Southern Economic Journal, vol. 65, pp. 603-10.

Isaac, R. M. and Plott, C. (1981) 'Price controls and the behavior of auction markets: an experimental examination', American Economic Review, vol. 71, pp.448-59.

Kruse, J. and Thompson, M. (2001) 'A comparison of salient rewards in experiments: money and class points', Economics Letters, vol. 74, pp. 113-17.

Lindbeck, A. (1972) The Political Economy of the New Left, New York: Harper and Row. Mansfield, E. and Yohe, G. (2000) Microeconomics: Theory and Application, 10th edn, New York:W.W. Norton.

Navarro, P. (1987) 'Rent control in Cambridge, Massachusetts', Public Interest, vol. 91, pp. 83-100.

O'Sullivan, A. and Sheffrin, S. (2001) Economics Principles and Tools, 2nd edn, Englewood Cliffs, NJ: Prentice Hall.

Perloff, J. M. (1999) Microeconomics, Reading, MA: Addison Wesley.

RAND Corporation (1970) 'The effects of rent control on housing in New York City', in Rental Housing in New York City: Confronting the Crisis, RM-6190-NYC, New York. Sharp, A., Register, C. and Grimes, P. (2002) Economics of Social Issues, 15th edn, New York: McGraw-Hill.

Smith, A. (1776) The Wealth of Nations, ed. Edwin Cannan, New York: Modern Library, 1937. Smith, V.L. (1982) 'Microeconomics systems as an experimental science', American Economic Review, vol. 72, pp. 923-55.

Smith, V.L. and Williams, A. (1981) 'On nonbinding price controls in a competitive market', American Economic Review, vol. 71, pp. 467-74.

Stiglitz, J. E. (2000) Economics of the Public Sector, 3rd edn, New York:W.W. Norton \& Co.

Varian, H. (1999) Intermediate Microeconomics: A Modern Approach, 5th edn, New York: W.W. Norton \& Co.

Wells, D. (1991) 'Laboratory experiments for undergraduate instruction in economics', Journal of Economic Education, vol. 22, pp. 293-300. 


\section{Notes}

1 Further readings on classroom experiments and how competitive markets clear are contained in DeYoung (1993) and Wells (1991).

2 See RAND Corporation (1970), Navarro (1987) and Gyourko and Linneman (1990) for a few studies that examine the effects of rent controls on landlords' maintenance expenditures.

3 This can be changed to examine some adjustment in other variables that affect demand and supply, such as side payments and black markets, to name two.

4 The instructions explain the conversion of experimental money to some reward medium (a number of options exist, such as quiz points, monetary reward for all participants, and random payouts to students). See Kruse and Thompson (2001) for a discussion of class points as salient rewards in experiments.

5 Several methods can be used to queue up the buyers. The instructor can randomly choose among the participants who want to rent at the available prices until none is left, or a prearranged queue can be used.

6 A new period begins with a new assignment of costs and rental budgets. We have chosen to reassign the rental budgets and costs each period so that over the course of the experiment, although the demand and supply schedules are relatively stable, no individual is stuck with a perennially low rental budget or high cost. The expected total reward (quiz points, money, etc.) is roughly equal at the end of eight periods. However, the instructor can choose to give the participants the same per period rental budget or cost - the market equilibrium prediction is the same.

7 The accurate demand and supply functions are step functions due to the discreteness of the market we have created. For instructional purposes, one can draw the more familiar continuous linear demand and supply curves by connecting the $(Q, P)$ coordinates of the demand and the supply schedules.

8 For an excellent introductory text on how to design and conduct good experiments, see Davis and Holt (1993).

\section{Acknowledgements}

Financial support from Texas Tech Teaching Learning and Technology Center Faculty Incentive Grant to Professor Jamie B. Kruse, Experimental Economics in the Classroom: Experimental Learning Modules is gratefully acknowledged. In addition, we thank two anonymous reviewers for their valuable comments and suggestions.

\section{Contact details}

Jamie Brown Kruse

Professor of Economics

East Carolina University

Greenville, North Carolina

USA

Ozlem Ozdemir

Assistant Professor of Economics

Yeditepe University

Istanbul, Turkey
Mark A.Thompson (correspondence author)

Assistant Professor of Economics

Stephen F. Austin State University

Department of Economics and Finance

P.O. Box 13009 - SFA Station

Nacogdoches, TX 75962

USA

tel: $\quad$ (936) 4681501

email: thompsonma@sfasu.edu 



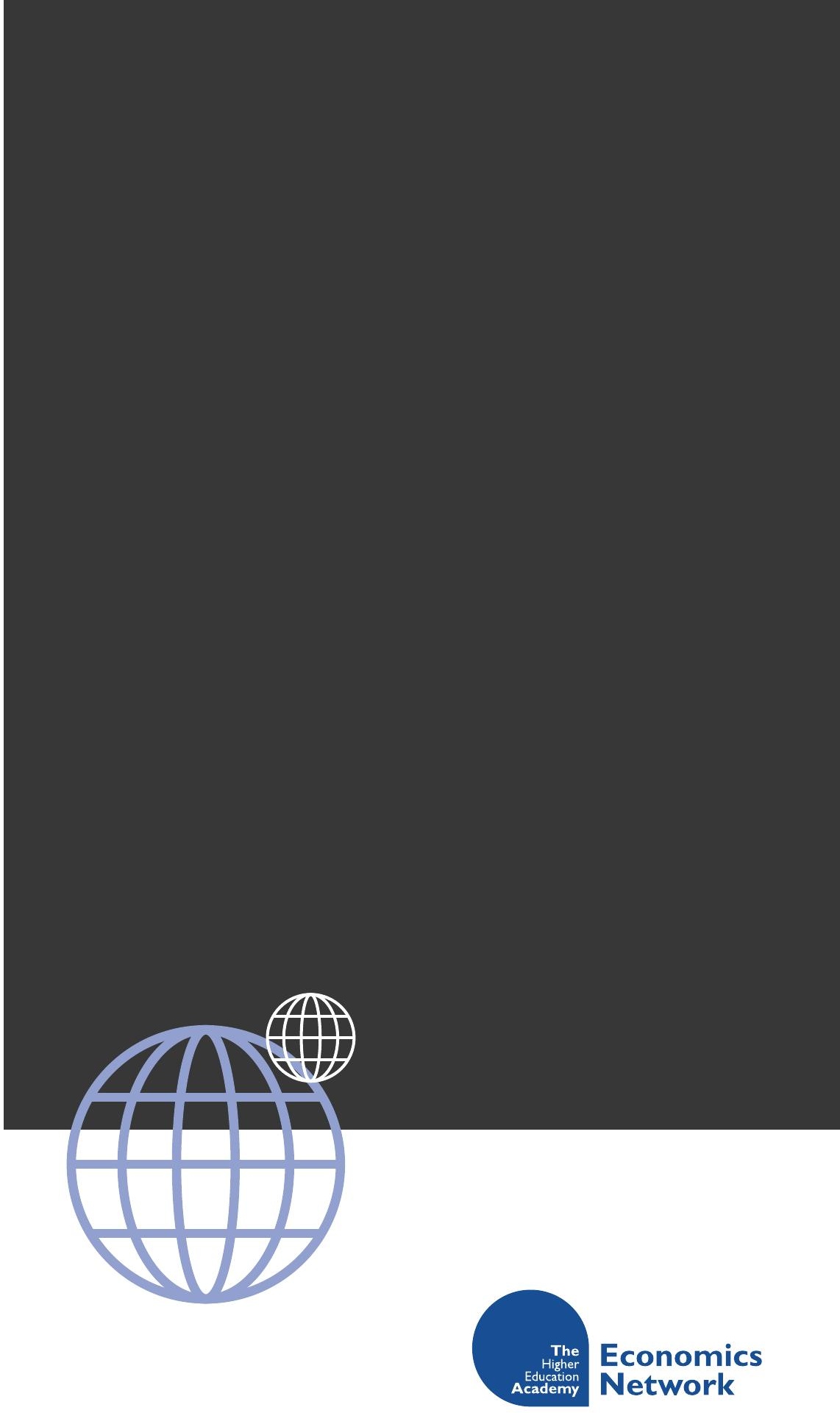

